\title{
SPURIOUS EFFECTS OF ELECTRON EMISSION FROM THE GRIDS OF A RETARDING FIELD ANALYSER ON SECONDARY ELECTRON EMISSION MEASUREMENTS. RESULTS ON A (111) COPPER SINGLE CRYSTAL
}

\author{
J. PILLON, D. ROPTIN and M. CAILLER \\ Laboratoire de Physique du Métal, Ecole Nationale Supérieure de Mécanique de Nantes \\ 3, rue du Maréchal Joffre 44041 Nantes Cedex, France
}

(Reçu le 10 mars 1976, révisé le 19 mai 1976, accepté le 2 juillet 1976)

\begin{abstract}
Résumé. - On étudie les effets parasites d'un système analyseur 4 grilles à champ retardateur dans les mesures d'émission d'électrons secondaires de faible énergie.

Leur comportement est étudié et les deux pics qui apparaissent dans le spectre énergétique sont interprétés comme résultant d'une émission tertiaire par les grilles. On montre qu'il est nécessaire de séparer le pic électronique secondaire vrai de ces pics parasites.

On donne le spectre et les rendements $\sigma$ et $\eta$ pour un cristal (111) de cuivre de propreté contrôlée par spectroscopie Auger.
\end{abstract}

\begin{abstract}
Spurious effects of a four grid retarding field analyser were studied for low energy secondary electron measurements. Their behaviour was investigated and two peaks in the energy spectrum were interpreted as resulting from tertiary electrons from the grids. It was shown that the true secondary electron peak has to be separated from these spurious peaks. The spectrum and the yields $\sigma$ and $\eta$ obtained for a $\mathrm{Cu}$ (111) crystal after a surface cleanness control by Auger spectroscopy are given.
\end{abstract}

1. Introduction. - Secondary electrons emitted with an energy of a few electron volts come from the surface atomic layers. The emission is thus very sensitive to the nature of the surface. The vacuum quality and surface preparation as controlled by Auger spectroscopy are then decisive tests of measurement quality. The progress in vacuum techniques and the development of investigation methods for surfaces provide more precise values for secondary electron emission. However there are still differences in the energy distribution of the true secondary electrons as given by different authors. Also theoretical studies [1] prove that we must consider, simultaneously all the observables of the phenomenon. With a view of reducing these differences we measured the energy distribution of the secondary electron emission of a copper single crystal (111). These studies, which were carried out with a retarding field Auger spectroscope showed that, in the low energy region of the spectrum, it was imperative to separate the spurious effects from the true phenomenon. If this is not done, the spectrum may be greatly distorded.

2. Apparatus and experimental procedures. - The measurement device is a standard 4 grid Vacuum Generators Auger spectroscope (Fig. 1). Baking at $300^{\circ} \mathrm{C}$ gave a $10^{-10}$ torr vacuum so introducing negligeable surface contamination by residual gases.

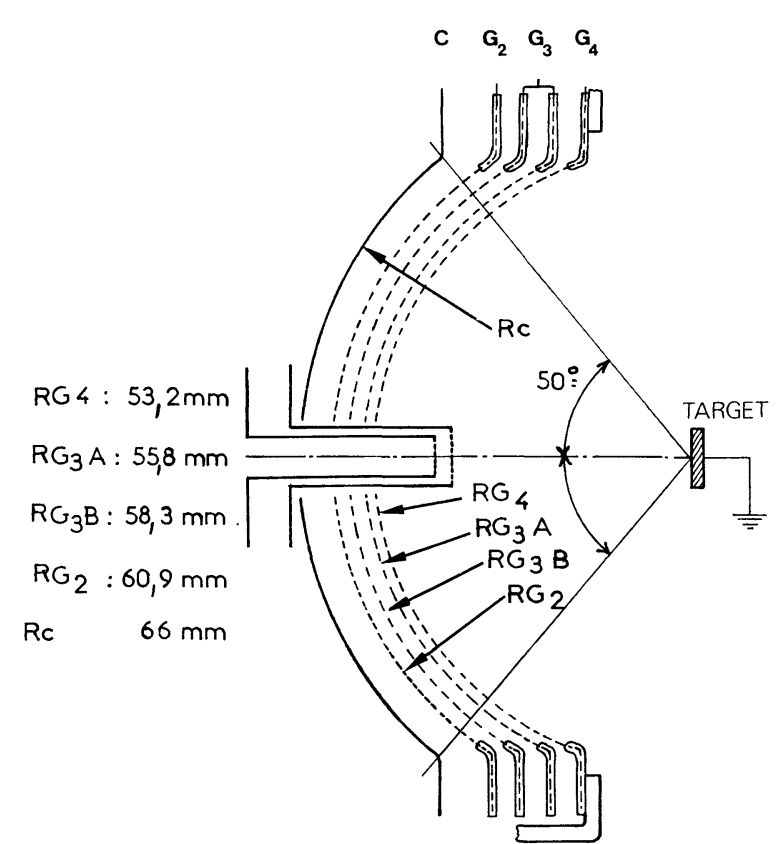

Fig. 1. - Four grid device.

The target is bombarded at normal incidence. The electron gun produces a primary electron beam accelerated with a variable voltage with energies from a few $\mathrm{eV}$ to $2500 \mathrm{eV}$ and with an intensity smaller than 
$2 \mu \mathrm{A}$. During the measurements, the variation of the primary current intensity is less than $1 \%$. The analysis system grids are made of stainless steel wires $0,04 \mathrm{~mm}$ in diameter $(31$ meshes per $\mathrm{cm})$, each of them having $75 \%$ transparency.

Usually the $G_{4}$ grid is earthed and in this case, ensures the existence of a free-field space around the target. A potential $V_{\mathrm{R}}$ is applied to the $\mathrm{G}_{3}$ grid allowing the passage of only those electrons with an energy $E_{\mathrm{S}}$ higher than $e V_{\mathrm{R}}$. In fact, in order to improve the apparatus resolution (up to $1.4 \%$ for a primary energy of $100 \mathrm{eV}$ ) the $G_{3}$ grid is split into $G_{3 A}$ and $\mathrm{G}_{3 \mathrm{~B}}$. The $\mathrm{G}_{2}$ grid is earthed eliminating tertiary electron emission by the collector. It also reduces the capacity coupling between $\mathbf{G}_{3}$ and the collector. The collector accelerates the electrons that have passed through the grid system. The energy distribution $N(E)$ and its derivative are obtained by modulating the $\mathrm{G}_{3}$ grid.

The target (6 $\mathrm{mm}$ in diameter and $1.5 \mathrm{~mm}$ thick) was sliced out by electro-erosion from single crystal bar of copper $(5 \mathrm{~N})$ oriented by the Lauë method. The analysed surface was mechanically polished with a $1 \mu$ diamond paste then electrolytically polished in a $66 \%$ orthophosphoric acid solution. After rinsing in distilled water and drying, the target was immediately placed in vacuum. The target undergoes an in-situ cleaning consisting of a series of argon ion sputtering (accelerating voltage : $430 \mathrm{eV}$, current density : $\left.2 \mu \mathrm{A} / \mathrm{cm}^{2}\right)$ and annealing $\left(400{ }^{\circ} \mathrm{C}\right)$ cycles. The surface cleanness is controlled by Auger spectroscopy. The spectrum shown (Fig. $2 A$ ) was obtained after three cycles. On the same figure we also present the complete energy spectrum for $E_{\mathrm{p}}=200 \mathrm{eV}$ (Fig. 2B).
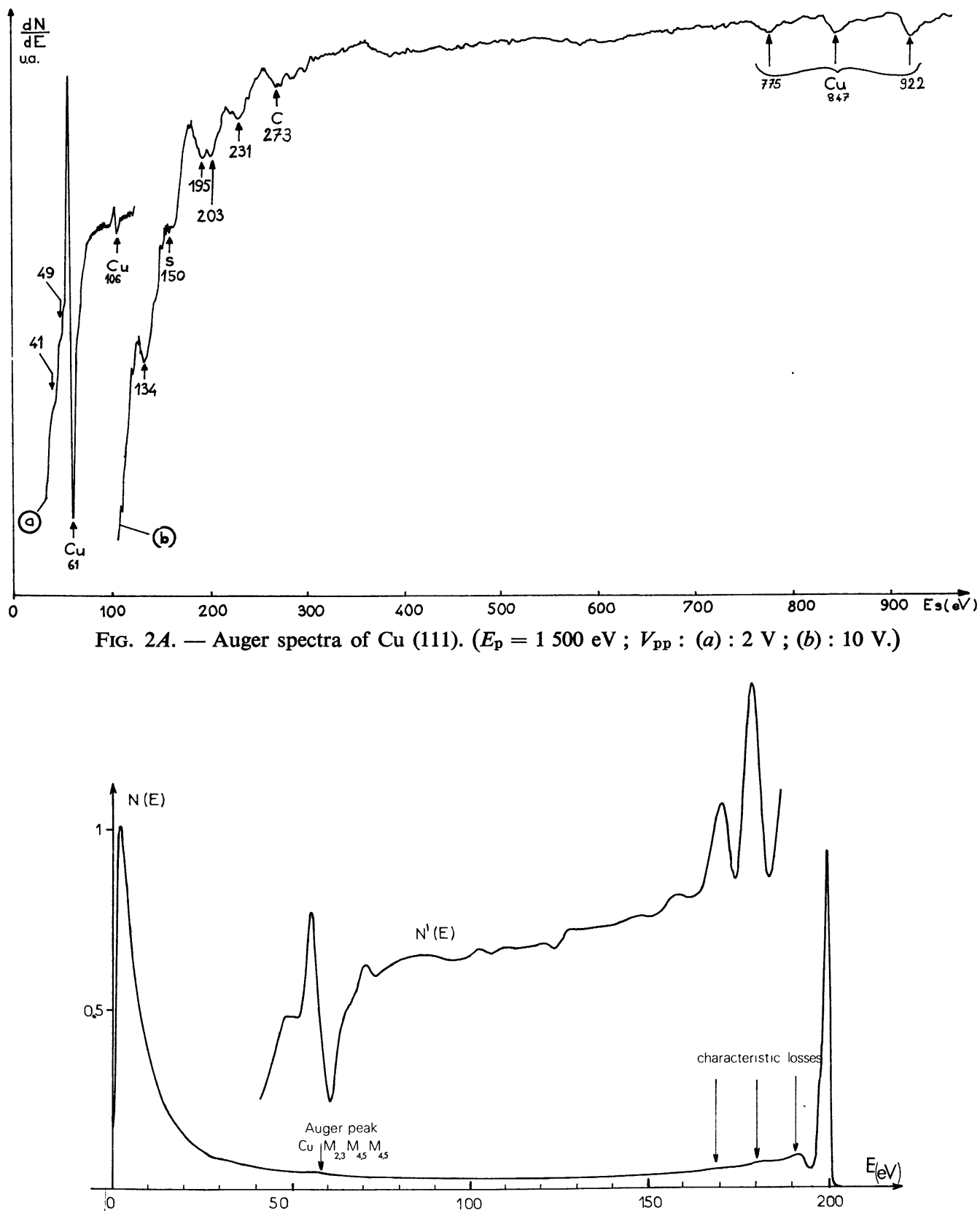

FIG. $2 B$. - Energy spectrum of $\mathrm{Cu}(111)\left(E_{\mathrm{p}}=200 \mathrm{eV}\right)$. 
3. Spurious grid effects. - The presence of parasite peaks in the energy distribution $N(E)$ was first reported by Morisson and Lander [2]. More recently, Wei et al. [3], Cross [4], Shimizu [5] reported similar observations. Considering the importance of these phenomena we measured the influence of the peaks on the energy distribution of the true secondary electrons. The measurements were made on a (111) crystal copper target before cleaning with a $300 \mathrm{eV}$ primary energy in a $2 \times 10^{-10}$ torr vacuum with a $30 \mathrm{mV}$ modulation voltage amplitude.
3.1 SHIFTS WITH THE VARIATION OF THE GRID voltAGES. - The normal spectrum $N(E)$ (Fig. 3) obtained for negative values of $V_{\mathrm{G}_{3}}\left(V_{\mathrm{G}_{3}}=-E\right)$ is completed by the signal obtained for positive values of this potential. The curve (1) was obtained for $V_{\mathrm{G}_{4}}=V_{\mathrm{G}_{2}}=0 \mathrm{~V}$ and has a positive peak at $V_{\mathrm{G}_{3}}=0.3 \mathrm{~V}$ and a negative peak at $V_{\mathrm{G}_{3}}=1.4 \mathrm{~V}$. On the same curve we observe that the negative peak is shifted when the $G_{2}$ voltage changes from $0 \mathrm{~V}$ to $15 \mathrm{~V}$ (spectra (2), (3), (4)), its extremum corresponding to $V_{G_{3}}=V_{G_{2}}+1.4 \mathrm{~V}$. The energy distribution of the

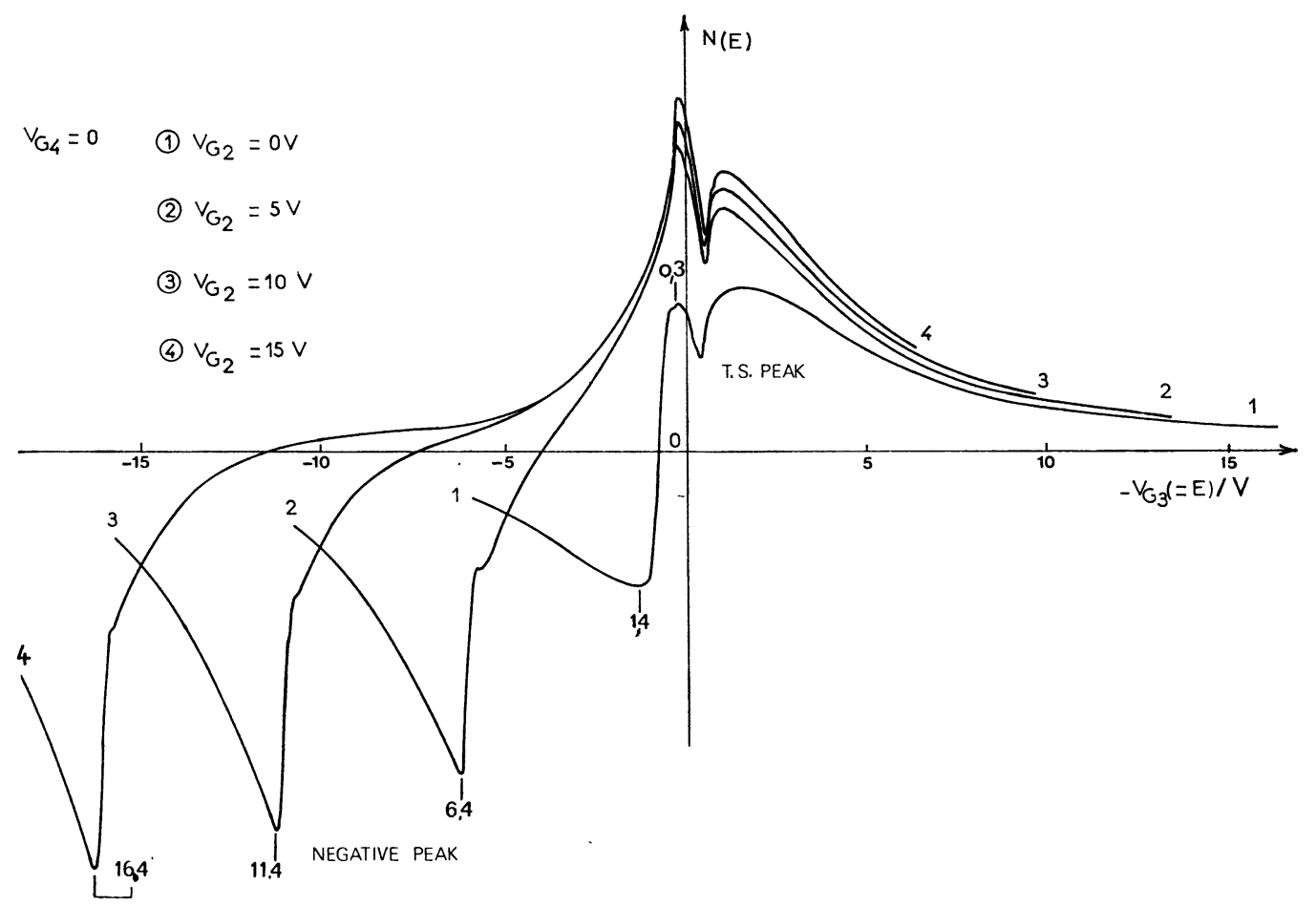

FIG. 3. - Shifts of the negative peak with $G_{2}$ grid voltages.

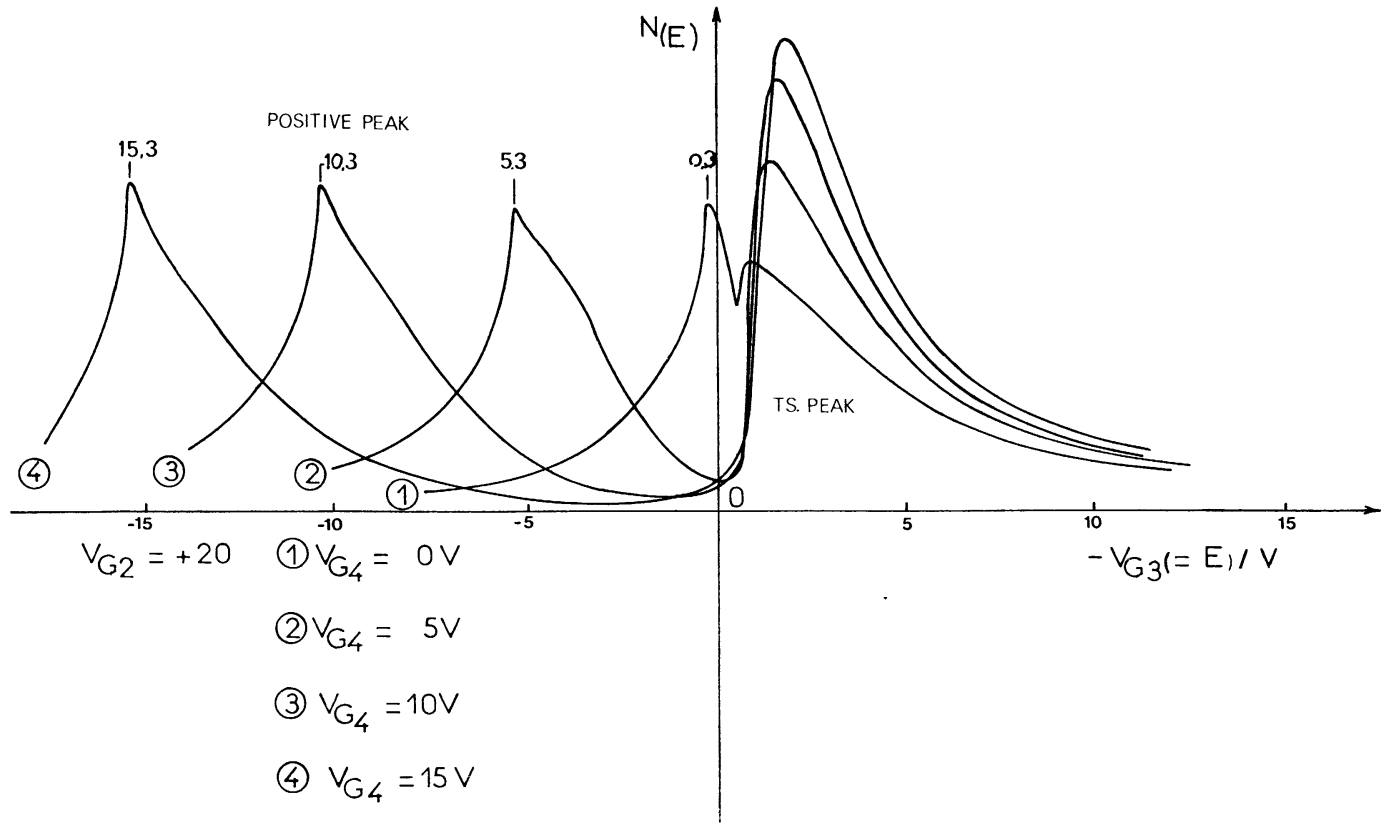

FIG. 4. - Shifts of the positive peak with $\mathrm{G}_{4}$ grid voltages. 
true secondary electrons is considerably modified when $V_{\mathrm{G}_{2}}$ varies from 0 to $5 \mathrm{~V}$. On the other hand, the extremum and the half height width do not change when $V_{\mathrm{G}_{2}}$ varies from 5 to $15 \mathrm{~V}$. The positive peak position also depends on $V_{\mathrm{G}_{4}}$ as shown on figure 4 obtained for $V_{\mathrm{G}_{2}}=20 \mathrm{~V}$. Then the peak position is given by $V_{\mathrm{G}_{3}}=V_{\mathrm{G}_{4}}+0.3 \mathrm{~V}$. The energy distribution is greatly modified only when $V_{\mathrm{G}_{3}}$ varies from 0 to $5 \mathrm{~V}$ (Curves (1) and (2)). The intensity of the distribution-maxima increases with $V_{\mathbf{G}_{4}}$, probably related to an increase of the collected current.

3.2 INTERPRETATION. - For positive values of $V_{\mathrm{G}_{3}}$, the positive and negative peaks express respectively an increase or a decrease of the collector current. Cross [4], using a 3 grid analyser, obtained only a positive peak and attributed these variations to tertiary emission of the grids. As for the presence and the shift of the two peaks, our observations confirm those of Wei et al. who attributed them to field penetration i. e. to a transparency variation of the filtering grid. On figure 5 , we reproduce the spectrum 3 from figure 4 and the corresponding variations with $V_{\mathrm{G}_{3}}$ of the grid currents $i_{\mathrm{G}_{4}}$ and $i_{\mathrm{G}_{2}}$. For $V_{\mathrm{G}_{3}}>0$, there is a good agreement between the decrease in $i_{\mathrm{G}_{4}}$, the increase in $i_{\mathrm{G}_{2}}$, and the peak position. That is to say a more important number of electrons going through $\mathrm{G}_{3}$ are able to reach $\mathrm{G}_{2}$ and the collector, increasing $i_{\mathrm{G}_{2}}$ and $I_{\mathrm{c}}$. From these measurements we were able to calculate $-\mathrm{d} i_{\mathrm{G}_{4}} / \mathrm{d} V_{\mathrm{G}_{3}}$ and $\mathrm{d} I_{\mathrm{c}} / \mathrm{d} V_{\mathrm{G}_{3}}$ and verify the agreement between these two quantities. In fact $\mathrm{d} I_{\mathrm{c}} / \mathrm{d} V_{\mathrm{G}_{3}}$ in the energy distribution and in this range corresponds to the positive peak.

Origin of the $i_{\mathrm{G}_{4}}$ variation. - Initially rejected by
Wei et al. on the ground of the relative importance of the parasite peaks compared with the true peak, the tertiary emission hypothesis was developed by Cross who compared the principal peak intensity $N_{1}(E)$ and the positive parasite peak intensity $N_{2}(E)$ and attributed the latter to the emission of grids $G_{3}$ and $\mathrm{G}_{4}$. In fact, as we have pointed out, the emission of grid $G_{3}$ generates the negative peak and does not contribute to the positive peak. Figure 6 describes the mecanisms of creation and shift of the different peaks for a four grid analyser. Potentials barriers $-e V_{G}$ are referenced to the target level. The creation of positive and negative peaks is described for positive values of $V_{\mathrm{G}_{4}}$ and $V_{\mathrm{G}_{2}}$ with $V_{\mathrm{G}_{2}}>V_{\mathrm{G}_{4}}$. On the schematic diagram the abscissa and the variable potential barrier $-e V_{\mathrm{G}_{3}}$ were put at the same level for three intermediate characteristic positions $1,2,3$ corresponding respectively to the recording of secondary, positive and negative peaks. The energy distribution of the secondary electron is obtained when $-\mathrm{eV}_{\mathrm{G}_{3}}$ is slightly higher than the target level (position 1). Filtering of tertiary electrons from grid $G_{4}$ by grid $G_{3}$ occurs when the variable barrier $-e V_{\mathrm{G}_{3}}$ is slightly higher than the constant barrier $-e V_{\mathbf{G}_{4}}$ (position 2). We can then explain the positive peak recording whose position is localized for $V_{\mathrm{G}_{3}}=V_{\mathrm{G}_{4}}$. A variation of this voltage gives an equal shift of the parasite peak. The filtering of tertiary electrons from $G_{3 A}$ and $G_{3 B}$ by grid $\mathrm{G}_{2}$ occurs when $-e V_{\mathrm{G}_{3}}$ becomes lower than $-e V_{\mathrm{G}_{2}}$ (position 3). Consequently the collector current decreases. The result is that the origin of the negative peak is determined by $V_{\mathrm{G}_{3}}=V_{\mathrm{G}_{2}}$.

The tertiary electrons emitted by the grid $\mathrm{G}_{2}$, under usual experimental conditions $\left(V_{\mathrm{C}}>V_{\mathrm{G}_{2}}\right)$, always

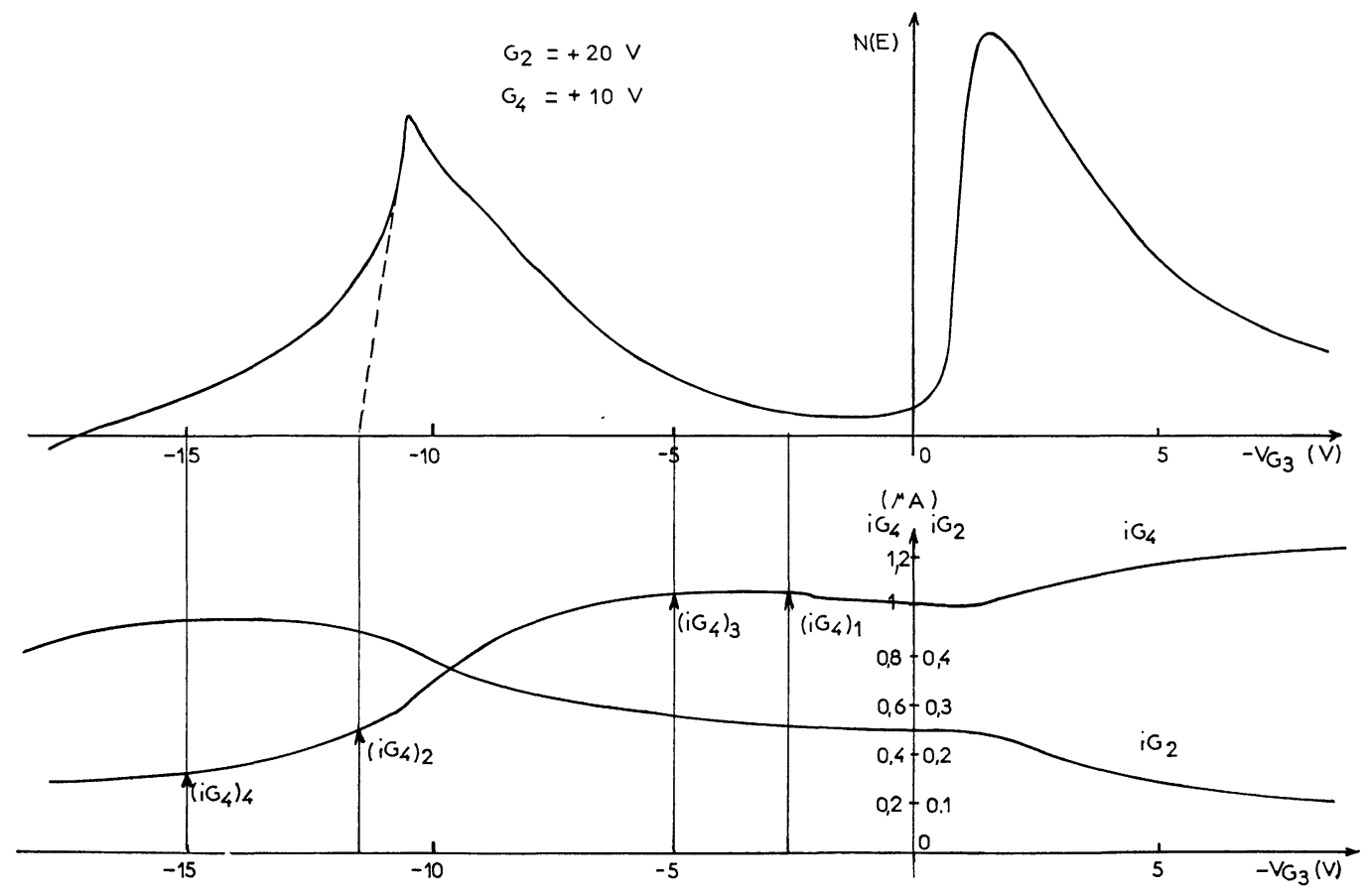

FIG. 5. - Variation of the currents on the grids $G_{4}$ and $G_{2}$. 


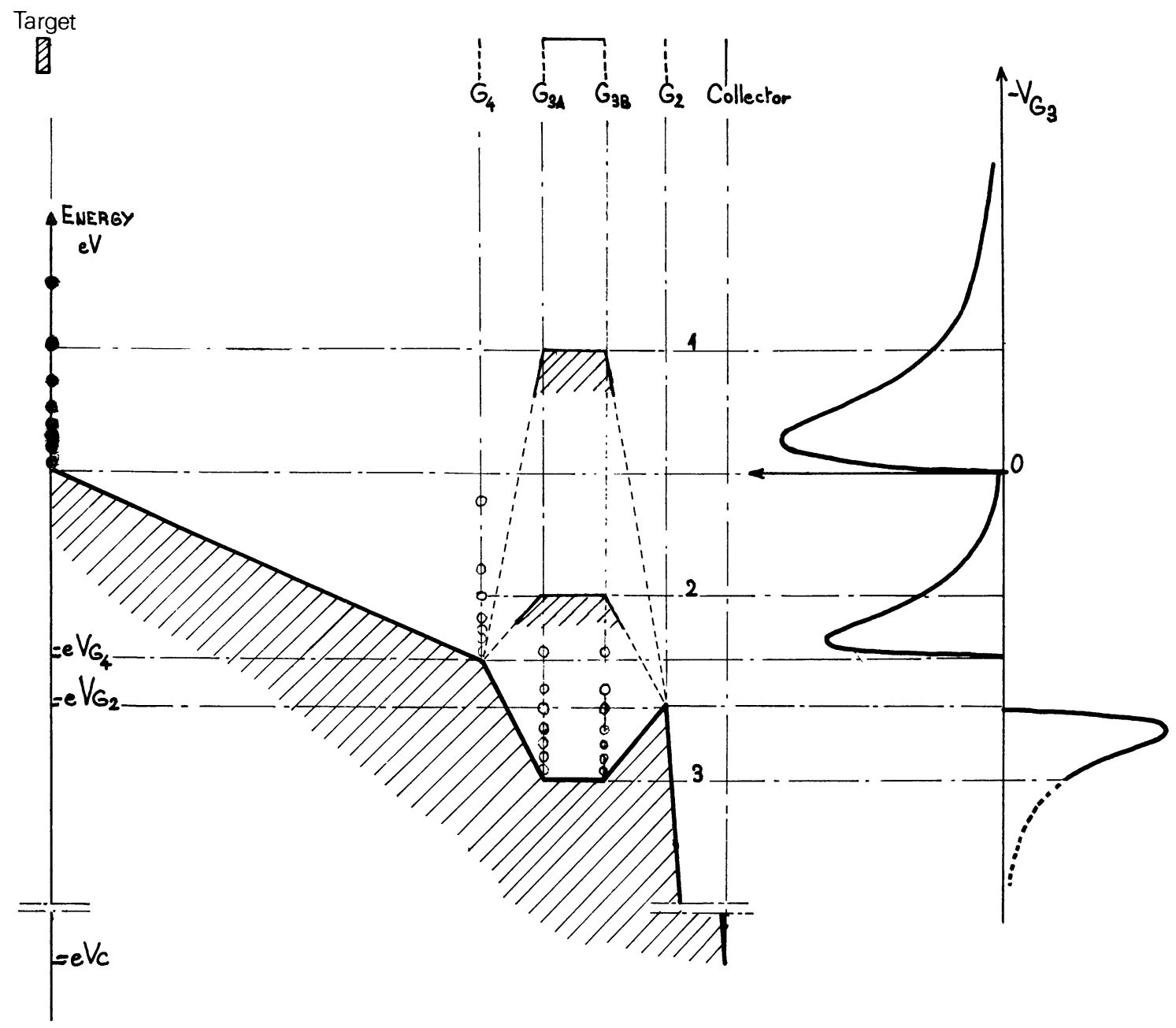

FIG. 6. - Filtering mecanism.

reach the collector and give an extra nearly constant direct contribution to the collector current. On figure 7, we show how positive and negative peaks interfere in the case where $-e V_{\mathrm{G}_{2}}>-e V_{\mathrm{G}_{4}}$. The filtering of the tertiary electrons from $G_{3}$ is the same as above. However, the tertiary electrons emitted by the grid $\mathrm{G}_{4}$ whose energy is less than $-e\left(V_{\mathrm{G}_{2}}-V_{\mathrm{G}_{4}}\right)$ do not reach the collector and the corresponding part of the positive peak disappears in agreement with reported experimental results (Fig. 8). In fact, on the grids there may be not only a tertiary emission but also different physical mechanisms such as elastical deflexion reducing the normal momentum of the secondary electrons. The quantitative importance of these effects is difficult to estimate. However, we can roughly determine the importance of the parasite peaks from the values $\left(i_{\mathrm{G}_{4}}\right)_{1}$ and $\left(i_{\mathrm{G}_{4}}\right)_{2}$ of the current $i_{\mathrm{G}_{4}}$ on each side of the positive peak (Fig. 5). Let $I_{\mathrm{S}}$ be the secondary current emitted by the target and $\alpha$ the transparency of grid $G_{4}$. The grid $G_{4}$ is reached by a current $(1-\alpha) I_{\mathrm{S}}$. For $V_{\mathrm{G}_{3}}-V_{\mathrm{G}_{4}} \sim-8 \mathrm{~V}$, only back scattered tertiary electrons have sufficient energy to leave the grid $G_{4}$. The corresponding current is $i_{t_{4}}^{b}$ and $\left(i_{\mathrm{G}_{4}}\right)_{1}=(1-\alpha) I_{\mathrm{S}}-i_{\mathrm{t}_{4}}^{b}$. If $V_{\mathrm{G}_{3}}-V_{\mathrm{G}_{4}}$ is slightly REVUE DE PHYSIQUE APPLIQUÉE. - T. 11, N 6, NOVEMBRE 1976 positive, with the hypothesis that all tertiary electrons emitted by $G_{4}$ are accelerated by $G_{3}$ towards the collector, the current emitted is $i_{\mathrm{t}_{4}}$ and $\left(i_{\mathrm{G}_{4}}\right)_{2}=(1-\alpha) I_{\mathrm{S}}-i_{\mathrm{t}_{4}}$. In our experimental conditions for copper, $\sigma \simeq 1.2$ and $\eta \simeq 0.3$ [5]. One quarter of the secondary current $I_{\mathrm{S}}$ emitted by the target comes from back scattered electrons with energies between 50 and $300 \mathrm{eV}$ and three quarters comes from true secondary electrons $\left(E_{\mathrm{S}}<50 \mathrm{eV}\right)$. If $V_{\mathrm{G}_{4}}=10 \mathrm{~V}$, these electrons are accelerated towards the grid $\mathrm{G}_{4}$ and reach it with mean energies of about 200 and $20 \mathrm{eV}$, respectively. $G_{4}$ is made of steel $(\sigma=0.3$ at $20 \mathrm{eV}$ and 1.2 at $200 \mathrm{eV}$ [6]), also $\eta=0.3$ at $200 \mathrm{eV}$ [7]. The total tertiary current emitted by the steel grid is :

$$
i_{\mathrm{t}_{4}}=\frac{3}{4}(1-\alpha) I_{\mathrm{S}} \cdot 0.3+\frac{1}{4}(1-\alpha) I_{\mathrm{S}} \cdot 1.2
$$

the backscattered tertiary current is :

$$
i_{\mathrm{t}_{4}}^{r}=\frac{1}{4}(1-\alpha) I_{\mathrm{s}} \cdot 0.3
$$

and

$$
\frac{i_{\mathrm{G}_{4}}(1)}{i_{\mathrm{G}_{4}}(2)} \sim 1.8
$$

Experimentally (Fig. 5) $\quad i_{\mathrm{G}_{4}}(1)=1.06 \quad \mu \mathrm{A} \quad$ and 


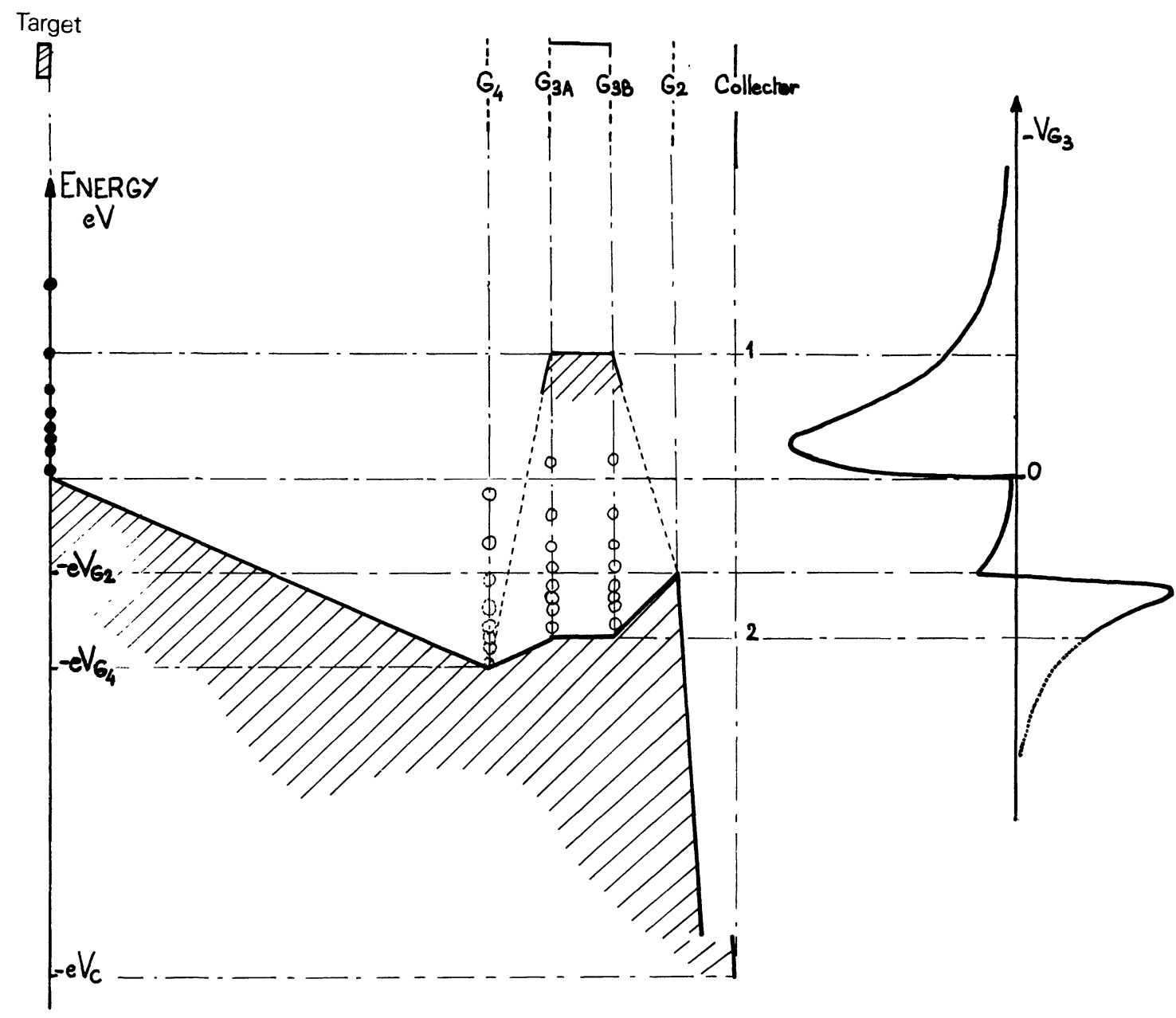

FIG. 7. - Mecanism of disappearance of the positive peak for $V_{\mathrm{G}_{4}}>V_{\mathrm{G}_{4}}$.

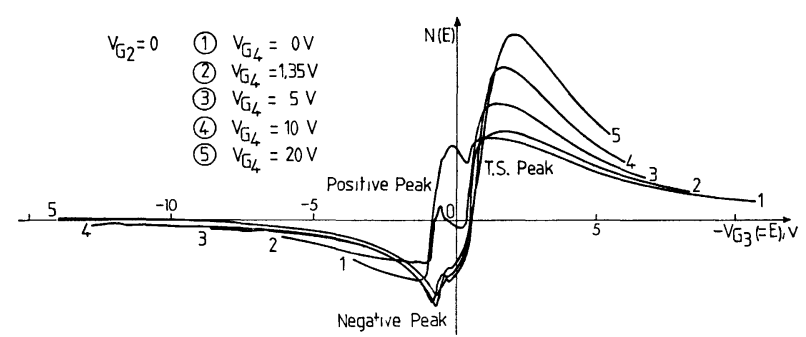

FIG. 8. - Results showing the disappearance of the positive peak for $V_{\mathrm{G}_{4}}>V_{\mathrm{G}_{2}}$.

$i_{\mathrm{G}_{4}}(2)=0.5 \mu \mathrm{A}$ giving nearly the same value for the ratio.

Transparency variation. - Wei et al. have interpreted the $i_{\mathrm{G}_{4}}$ current variations as due to the transparency variation of $\mathrm{G}_{4}$ when the field behind this grid goes from an accelerating to a retarding state. To explain the measured variations of $\mathrm{G}_{4}$ this transparency must vary from $63 \%$ to $87 \%$. The field penetration and the corresponding change in the trajectories of low energy electrons may be the cause of this transparency variation, but this hypothesis hardly accounts for the dissymetric shape of the parasite peaks, similar in fact, to the shape of the secondary electron energy distributions. In the same way, the field penetration cannot explain the attenuation and the suppression of the positive parasite peak when $V_{\mathrm{G}_{4}}$ becomes higher than $V_{\mathrm{G}_{2}}$ (Fig. 8), whereas in our interpretation, this suppression is an immediate consequence of the filtering of tertiary electrons from grid $G_{4}$ by grid $G_{2}$. Finally, when the primary energy $E_{\mathrm{p}}$ varies from $50 \mathrm{eV}$ to $1500 \mathrm{eV}$, we observed that the parasite peak amplitude was maximum for $E_{\mathrm{p}}=300-400 \mathrm{eV}$ where the secondary emission yield of steel is maximum [6].

3.3 CHOICE OF $\mathrm{G}_{4}$ AND $\mathrm{G}_{2}$ voltages. - When $V_{\mathrm{G}_{4}}$ or $V_{\mathrm{G}_{2}}=0$ we observed an important dependance of the maximum position and half height width of the energy distribution in relation to the nearness of positive (or negative) peak. To separate the parasite peaks from the principal peak during the recording, we choose to apply a sufficient positive voltage to grids $G_{2}$ and $G_{4}$. But this voltage must be sufficiently low so as not to modify greatly the secondary electron trajectories. Also the current collected by grid $\mathrm{G}_{4}$ increases quickly, doubling when $V_{\mathrm{G}_{2}}$ increases from 0 to $20 \mathrm{~V}$. This increase is due to a growth of the true 
collection angle for secondary electrons which are accelerated towards grid $\mathrm{G}_{4}$. It is not a major drawback in itself but simultaneously an increasing number of low energy secondary electrons are stopped by grid $\mathrm{G}_{4}$, developing a risk of increased disturbance in the measurements. So, on figure 9, we show that the secon-

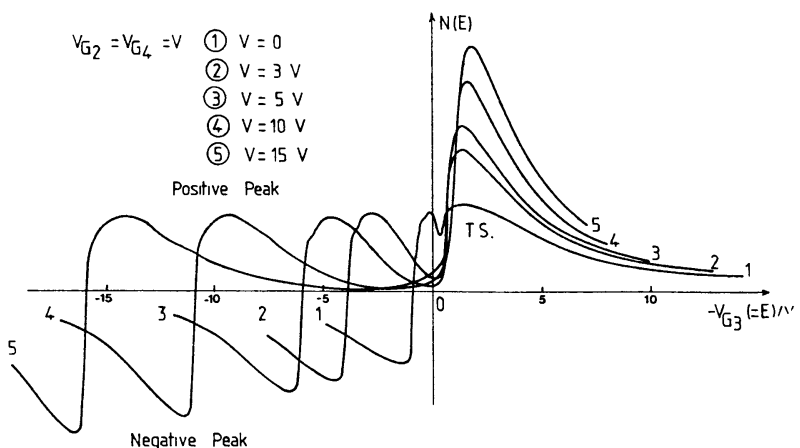

Fig. 9. - Shifts as a function of voltage on the grids $\mathrm{G}_{2} \mathrm{G}_{4}$

dary peak shifts towards higher energies, when $V_{\mathrm{G}_{4}}$ increases. Thus, it is important to keep the $V_{\mathrm{G}_{2}, \mathrm{G}_{4}}$ voltage as low as possible. To make the best choice we drew several energy spectra for $V_{\mathrm{G}_{2}, \mathrm{G}_{4}}=0 ; 3 ; 5$; 10 and $15 \mathrm{~V}$. Simultaneously the positive and negative peaks are shifted and to measure the influence of this shift we give in table I the values of the maximum position $E_{\max }$ and the half height width $E_{1 / 2}$ for the secondary peak.

\section{TABLE I}

$\begin{array}{llllll}\quad V_{\mathrm{G}_{2} / \mathrm{G}_{4}} \text { in volts } & 0 & 3 & 5 & 10 & 15 \\ E_{\max } \text { in } \mathrm{eV} & 1.4 & 0.9 & 0.8 & 1 & 1.2 \\ E_{1 / 2} \text { in } \mathrm{eV} & 5.7 & 4.1 & 3.8 & 3.6 & 3.6\end{array}$

We finally chose a voltage of $10 \mathrm{~V}$ because the characteristic values of the secondary peak do not vary strongly when $V_{\mathrm{G}_{2}, \mathrm{G}_{4}}$ varies from 5 to $15 \mathrm{~V}$.

4. Results on a (111) single-crystal copper target. We measured energy distributions and $\sigma, \eta, \delta$ yields for a (111) copper target after Auger control of the surface cleanness.

4.1 ENERGY Distribution (Fig. 10). - The results presented were obtained with $V_{\mathrm{G}_{2}, \mathrm{G}_{4}}=10 \mathrm{~V}$, for a primary current $i_{\mathrm{p}} \sim 2 \mu \mathrm{A}$ and a primary energy of $300 \mathrm{eV}$. The maximum of the true secondary electron distribution occurs at $V_{\mathrm{G}_{3}}=-2 \mathrm{~V}$. The zero value is obtained for $V_{\mathrm{G}_{3}}=-0.3 \mathrm{~V}$ and corresponds to the difference between the target and the grid $\mathrm{G}_{3}$ work functions. Our results are compared (Fig. 11) with different other results $[8,9,10]$ for a normalized value of maximum amplitude. Except for Scheibner and Tharp all these curves are narrow. This difference may be attributed to the spurious effect of the grids

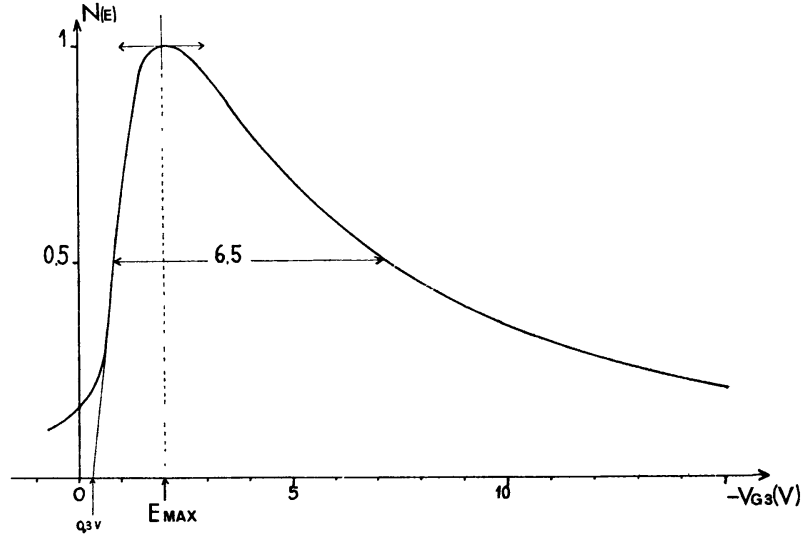

Fig. 10. - True secondary spectrum of $\mathrm{Cu}(111)\left(E_{\mathrm{p}}=300 \mathrm{eV}\right)$.

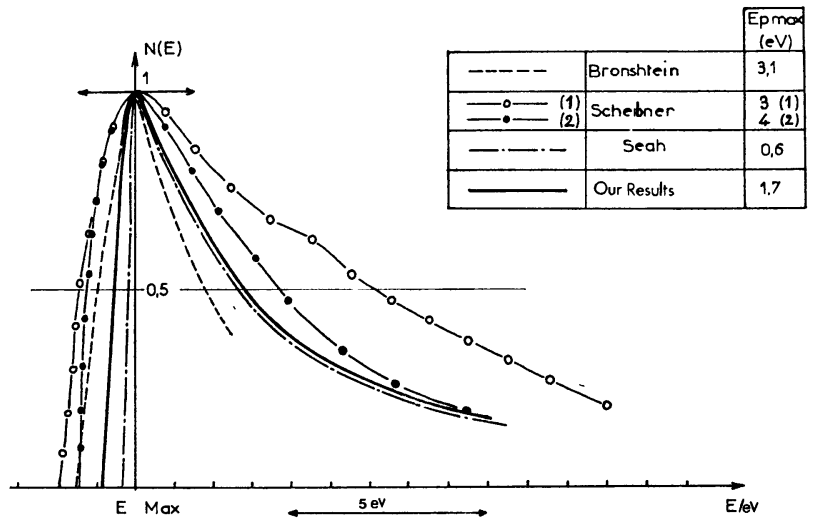

FIG. 11. - True secondary spectra obtained by other authors for copper.

because their apparatus was similar to ours with $V_{\mathrm{G}_{2}, \mathrm{G}_{4}}=0$. We observed that the curve width increased by $50 \%$ when $V_{\mathrm{G}_{2}, \mathrm{G}_{4}}$ goes from $10 \mathrm{~V}$ to $0 \mathrm{~V}$ and gives the energy distribution of Scheibner [2] as reported by Amelio. The results obtained by Bronshtein and Seah on polycrystalline copper targets have a comparable half height width but rather different maximum positions. However Seah used a $70^{\circ}$ oblique incidence and collected the secondary electrons in a very narrow solid angle (12 millisteradians). For $E>E_{\max }$ our peak has a shape similar to Seah's and may be described by a power law

$$
N(E) \propto \frac{1}{(E+4.5)^{1.6}} \frac{1}{(E+0.35)}
$$

but differs for $E<E_{\max }$ with a more rapid increase and then a shift of the maximum position. However, Seah noticed that for higher angles of emergence, the secondary peak moves to higher energies (for silver he reported a $50 \%$ variation when the angle of emergence varies from 0 to $60^{\circ}$ ).

We can conclude that our results and Seah's are similar and that the differences are due to different experimental conditions. 
4.2 Yields. - For one primary electron, the target emits $\sigma$ secondary electrons with $\delta$ electrons having an energy smaller than $50 \mathrm{eV}$ and $\eta$ electrons having an energy greater than $50 \mathrm{eV}$. To evaluate $\sigma$ we must measure the total secondary current $i_{\mathrm{s}}$ and the primary current $i_{\mathrm{p}}$. In fact, we measure the target current $i_{\mathrm{c}}$ and $i_{\mathrm{s}}$,

$$
\sigma=\frac{i_{\mathrm{s}}}{i_{\mathrm{p}}}=\frac{i_{\mathrm{s}}}{i_{\mathrm{s}}+i_{\mathrm{c}}}
$$

To attract all electrons towards the collector suppressing the charge of the fluorescent layer and the tertiary emission of the collector, we applied the same potential to the 4 grids and to the collector and measured the total secondary current in the collector.

We applied $\mathrm{a}+200 \mathrm{~V}$ potential, because for this value, the measured current $i_{\mathrm{s}}$ was more stable for $\mathrm{a} \pm 50 \mathrm{~V}$ variation around this value. Palmberg [11] with a 3 grid system suggested using a $300 \mathrm{~V}$ post acceleration. Under these conditions, the total yield curve $\sigma\left(E_{\mathrm{p}}\right)$ is to within experimental uncertainty, in good agreement with Bronshtein's results [6] (Fig. 12). To determine $\eta$ we measure the current $i_{\mathrm{s}}^{\prime}$ reaching the

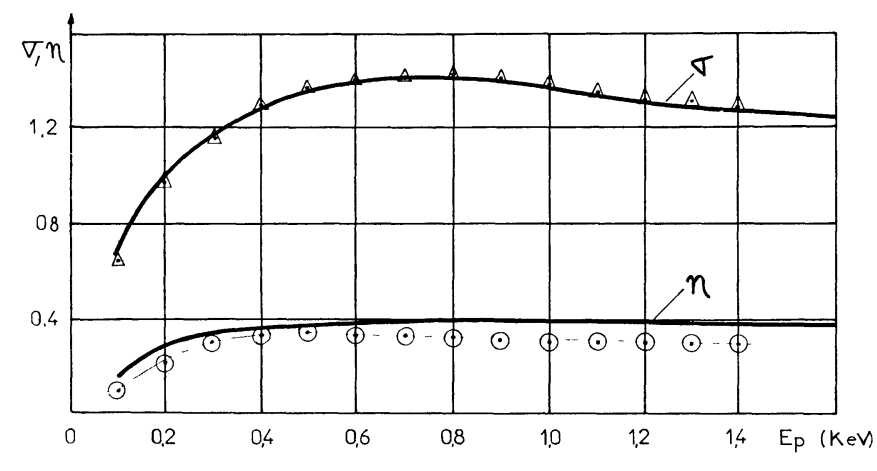

FIG. 12. - Secondary yields $\sigma$ and $\eta$ of copper. Bronshtein :

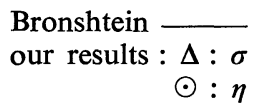

collector after passing through the filtration system, for a retarding potential of $50 \mathrm{~V}$. The $i_{\mathrm{p}}$ current is determined as above and if $\tau$ is the analyser transparency

$$
\eta=\frac{1}{\tau} \frac{i_{\mathrm{s}}^{\prime}}{i_{\mathrm{s}}+i_{\mathrm{c}}}
$$

To evaluate $\tau$ we used Richard's method [12] which is applied with a 2 grid analyser to give each measurement the transparency value of the grids. The identification with our system was obtained by connecting $\mathrm{G}_{2}$ to the collector and by applying the retarding potential to grid $\mathrm{G}_{3}$. The values obtained for $\eta$ are compared on figure 12 with Bronshtein's results [13]. Our results correspond to a collecting angle of $100^{\circ}$ and it is not surprising that our values are smaller than those of Bronshtein. For a cosinusoïdal distribution of secondary electrons, the corrective factor should be approximatively 1.65 whereas the experimental corrective factor is 1.5 for a $500 \mathrm{eV}$ primary energy. We must remark that the measured current includes tertiary electrons emitted by grid $\mathrm{G}_{3}$ as well as secondary electrons filtered by the same grid. Also the determination of the transparency of grids $G_{4}$ and $G_{3}$ is only approximate and does not take into account the secondary electron energies or an eventual transparency variation when grid $\mathrm{G}_{3}$ goes from 0 to $-50 \mathrm{~V}$. These first measurements have enabled a check to be made of the values of Bronshtein. The values of $\delta$ are obtained by substracting $\eta$ from $\sigma$.

Results for the energy spectra obtained with a cylindrical mirror analyser (CMA) [14] and correlated with image contrasts in a scanning electron microscope (SEM) are quite different from our results.

The origin of the disagreement is still unknown but some remarks may be made :

1) SEM image contrast seems to be related more to variations of the emission intensity that to the emission intensity itself.

Then an image without contrast will be an image in which each point of the surface has an equal emission intensity whatever the energy spectrum of the target.

The presence of impurities on the surface, resulting, for instance, from diffusion by local heating or others processes creates surface heterogeneities leading to contrasted images in SEM.

2) The 4 grid analysis system collects all the electrons emitted from bombardement of the surface whereas the CMA collects only in a finite solid angle. It is thus possible that the two collected distributions differ without either being in error.

3) In the same way CMA with its subsequent electron multiplier has a gain equal to $E . K(E)$ where $K(E)$ is the multiplier gain. This gain is higher for high energies than for low energies. The situation is different for a 4 grid analyser which gives $N(E)$ directly.

5. Conclusion. - The object of this work was to adapt a 4 grid retarding field analysis system to secondary electron emission studies. Such a device enables a control to be made of the cleanness of the analysed surface by Auger spectroscopy and hence provides precise information concerning the nature of the target. But if all necessary precautions are not taken, this system produces spurious effects which we have studied in detail. We showed that the origin of these perturbations is related to grid emission due either to an elastic reflection of secondary electrons on the grids in which they lose normal momentum or to a true tertiary electron emission from the grids under secondary bombardment. We proposed a means of separating the true and the spurious peaks. We thus obtained the energy distribution for secondary electrons emitted by a (111) copper single-crystal. The discrepancy in the experimental results may be due to the spurious effects on the grids. 
Acknowledgments. - The authors would like to thank MM. R. Bindi, H. Lanteri and Professor P. Keller of the laboratoire de Physique Expérimentale de la Faculté des Sciences de Nice, and M. Ganachaud of the Laboratoire de Physique du Métal de l'E. N. S. M. for numerous and fruitful discussions.

\section{References}

[1] Moulin, B., Ganachaud, J. P. et Cailler, M., Phys. Stat. Solids (b) 59, (1973), 79.

[2] Morrisson, J. et Lander, Bull. Amer. Phys. Soc. 11 (1968) 945.

[3] Wei, P. S. P., Cho, A. Y. et Caldwell, C. W., Rev. Sci. Instrum. 40 (1969), 1075.

[4] Cross, J. A., J. Phys. D 6 (1973), 622.

[5] Koshikawa, T. et Shimizu, R., J. Phys. D 6 (1973), 1369.

[6] Bronshtein, I. M. et Frajman, B. S. Emission Electronique Secondaire (Editions Naouka, Moscou), 1969, p. 343.

[7] Sternglass, E. J., Phys. Rev. 95 (1954) 345.
[8] Bronshtein, I. M. et ShChVChInskiy, Y. M., Radioteckhi Electr. 9 (1964), 738.

[9] Scheibner, E, J. et Tharp, L. N. Surf. Sci. 8 (1967) 247. [10] SEAH, M. P. Surf Sci. 17 (1969) 132.

[11] Palmberg, P. W. J. Appl. Phys. 38 (1967) 2137.

[12] Richard, C. Thèse $3^{\mathrm{e}}$ cycle, Université de Nice (1974).

[13] Bronshtein, I. M., Stozharov, V. M. et Pronin, V. P. Fiz. Tver. Tel. 13 (1971) 3359.

[14] Massignon, D., Le Gressus, C., Sopizet, R. et Durand, J. P. C. R. Hebd. Scéan. Acad. Sci. 281 (1975) 579. 